QUARTERLY OF APPLIED MATHEMATICS

VOLUME LXIX, NUMBER 3

SEPTEMBER 2011, PAGES 603-612

S 0033-569X(2011)01260-1

Article electronically published on April 26, 2011

\title{
DYNAMIC PATTERN FORMATION IN SWIFT-HOHENBERG EQUATIONS
}

\author{
BY \\ TUNG HOANG (Department of Mathematics, State University of New York at Buffalo, New York) \\ AND \\ HYUNG JU HWANG (Department of Mathematics, Pohang University of Science and Technology, \\ Pohang 790-784, Korea)
}

\begin{abstract}
In this paper we study nonlinear instability in the Swift-Hohenberg equation. The short-time pattern formation under the fully nonlinear setting is rigorously characterized. For unstable constant equilibria, we investigate the nonlinear dynamics of patterns which occur within the time interval of existence.
\end{abstract}

1. Introduction and main result. In this paper we study the problem of nonlinear pattern formation in the Swift-Hohenberg ( $\mathrm{SH}$ ) equation. The equation was derived in the context of thermal convection in [8. The SH equation has been used to model patterns in a variety of fluids and biological materials [3, 2] such as Taylor-Couette flow and lasers. Related works are found in 99, [10, 11], [13]. In this article, we will rigorously establish bounds on the short-time common patterns under the fully nonlinear regime and characterize the nonlinear patterns by a finite number of the maximal growing modes over a time scale of $\ln \frac{1}{\delta}$.

We consider the generalized Swift-Hohenberg equation

$$
\frac{d u}{d t}=\alpha u-(1+\triangle)^{2} u-f(u)
$$

on $\mathbb{T}^{d}=\left(0, L_{1}\right) \times\left(0, L_{2}\right) \times \ldots \times\left(0, L_{d}\right)$. Here $\alpha>0$ is a given constant, $f(\cdot)$ is a given function, and $f$ satisfies $f(0)=f^{\prime}(0)=0$. We impose the following boundary conditions and initial condition

$$
u=0, \quad \frac{\partial^{2} u}{\partial x_{i}^{2}}=0, \quad \frac{\partial^{4} u}{\partial x_{i}^{4}}=0 \quad \text { for all } i=1,2, \ldots, d
$$

at $x_{i}=0, L_{i}$ and

$$
u(x, 0)=u_{0}(x)
$$

where $u_{0}(x)$ is given.

Received March 24, 2010.

1991 Mathematics Subject Classification. Primary 35K50, 35B35, 92C15.

E-mail address: thoang@buffalo.edu

E-mail address: hjhwang@postech.ac.kr 
We use the norm in $L^{2}$ : for every $w(x) \in L^{2}\left(\mathbb{T}^{d}\right)$.

Notation 1.

$$
\|w\|=\|w\|_{L^{2}} .
$$

The corresponding linearized equation then takes the form

$$
\frac{d u}{d t}=(\alpha-1) u-\triangle^{2} u-2 \triangle u .
$$

Let $\mathbf{q}=\left(q_{1}, q_{2}, \ldots, q_{d}\right) \in \Omega=(\mathbb{N} \cup\{0\})^{d}$ and let

$$
e_{\mathbf{q}}(x)=\prod_{i=1}^{d} \sin \frac{q_{i} \pi x_{i}}{L_{i}}
$$

Then we have

$$
\left\|e_{\mathbf{q}}\right\|=\sqrt{\frac{L_{1} L_{2} \ldots L_{d}}{2^{d}}}
$$

and $\left\{e_{\mathbf{q}}\right\}$ forms a basis of the space of functions in $\mathbb{T}^{d}$ which satisfy (1.2).

We will solve the linearized equation (1.4) with the boundary and initial conditions.

$$
\begin{aligned}
& \frac{d u}{d t}=(\alpha-1) u-\triangle^{2} u-2 \triangle u, \\
& u=\frac{\partial^{2} u}{\partial x_{i}^{2}}=\frac{\partial^{4} u}{\partial x_{i}^{4}}=0, \quad \text { at } x_{i}=0, L_{i}, \quad \text { for all } i=1,2, \ldots, d, \\
& u(x, 0)=u_{0}(x) .
\end{aligned}
$$

Suppose the initial datum has the following decomposition:

$$
u_{0}(x)=\sum_{\mathbf{q} \in \Omega} w_{q} e_{\mathbf{q}}(x) .
$$

First we will find a solution with initial datum $u(x, 0)=e_{\mathbf{q}}(x)$. We seek a solution of the following form:

$$
u(x, t)=e^{\lambda t} e_{\mathbf{q}}(x)
$$

Then we have

$$
\left\{\begin{array}{l}
\frac{d u}{d t}=\lambda e^{\lambda t} e_{q}(x), \\
\triangle u=-q^{2} e^{\lambda t} e_{q}(x), \\
\triangle^{2} u=q^{4} e^{\lambda t} e_{q}(x),
\end{array}\right.
$$

where $q=\sqrt{\sum_{i=1}^{d}\left(\frac{q_{i} \pi}{L_{i}}\right)^{2}}$. Then $u(x, t)$ defined in (1.5) is a solution of (1.4) if and only if

$$
\lambda=\alpha-1-q^{4}+2 q^{2}=\alpha-\left(q^{2}-1\right)^{2} .
$$

Define $\lambda_{\mathbf{q}}=\alpha-\left(q^{2}-1\right)^{2}$. Then we will make the following assumption.

Assumption 1. There exists $\mathbf{q} \in \Omega$ such that $\lambda_{\mathbf{q}}>0$.

Note that if $L_{i}=k \pi, k \in \mathbb{N}$ for some $i$, then for $\mathbf{q}=\left(q_{1}, q_{2}, \ldots, q_{d}\right)$ with $q_{i}=k$, $q_{j}=0$, for all $j \neq i, q^{2}=\sum_{j=1}^{d}\left(\frac{q_{j} \pi}{L_{j}}\right)^{2}=1$. 
It is easy to see that there exist only finitely many values $\mathbf{q} \in \Omega$ such that $\alpha-\left(q^{2}-1\right)^{2}>$ 0 . Define

$$
\begin{gathered}
\lambda_{\max }=\max _{\mathbf{q} \in \Omega} \lambda_{\mathbf{q}}>0, \\
\Omega_{\max }=\left\{\mathbf{q} \in \Omega \mid \lambda_{\mathbf{q}}=\lambda_{\max }\right\},
\end{gathered}
$$

where $\nu>0$ is the gap between $\lambda_{\max }$ and the rest of $\lambda_{\mathbf{q}}, \mathbf{q} \in \Omega$. For $u_{0}=\sum_{\mathbf{q} \in \Omega} w_{\mathbf{q}} e_{\mathbf{q}}(x)$ we define

$$
\begin{gathered}
L\left[u_{0}\right](x, t)=\sum_{\mathbf{q} \in \Omega} w_{\mathbf{q}} e^{\lambda_{\mathbf{q}} t} e_{\mathbf{q}}(x), \\
M\left[u_{0}\right](x, t)=\sum_{\mathbf{q} \in \Omega_{\max }} w_{\mathbf{q}} e^{\lambda_{\max } t} e_{\mathbf{q}}(x) .
\end{gathered}
$$

Then $L\left[u_{0}\right](x, t)$ is a solution of (1.4), (1.2), (1.3).

We will frequently use the following ingredients: For $d \leq 3$ by the Sobolev imbedding theorem there exists a constant $C_{0}>0$ such that for all $g \in H^{2}\left(\mathbb{T}^{d}\right)$ we have

$$
\|g\|_{\infty\left(\mathbb{T}^{d}\right)} \leq C_{0}\|g\|_{H^{2}\left(\mathbb{T}^{d}\right)} .
$$

We assume that $f \in \mathbb{C}^{2}$ near 0 so that there exists $\eta>0$ such that

$$
C_{\eta}=\sup _{\|w\|_{\infty} \leq C_{0} \eta}\|f(w)\|_{C^{2}}<+\infty .
$$

From (1.9) and (1.10) we have

$$
\sup _{\|w\|_{H^{2}} \leq \eta}\|f(w)\|_{C^{2}} \leq C_{\eta}<+\infty .
$$

Then we have the following lemma.

Lemma 1. Let $L\left[u_{0}\right](x, t)$ and $\lambda_{\max }$ be defined in (1.8) and (1.6) respectively. Then we have

$$
\left\|L\left[u_{0}\right](x, t)\right\| \leq e^{\lambda_{\max } t}\left\|u_{0}\right\|
$$

Proof. We have

$$
L\left[u_{0}\right](x, t)=\sum_{q \in \Omega} w_{\mathbf{q}} e^{\lambda_{\mathbf{q}} t} e_{\mathbf{q}}(x) .
$$

Since the $e_{\mathbf{q}}(x)$ 's are orthogonal in $L^{2}\left(\mathbb{T}^{d}\right)$, we get

$$
\left\|L\left[u_{0}\right](x, t)\right\|^{2}=\sum_{\mathbf{q} \in \Omega} w_{\mathbf{q}}^{2} e^{2 \lambda_{\mathbf{q}} t}\left\|e_{\mathbf{q}}\right\|^{2},
$$

where $\lambda_{\mathbf{q}} \leq \lambda_{\max }$ and so we have

$$
\left\|L\left[u_{0}\right](x, t)\right\|^{2} \leq \sum_{\mathbf{q} \in \Omega} w_{\mathbf{q}}^{2}\left\|e_{\mathbf{q}}\right\|^{2} e^{2 \lambda_{\max } t}=\left\|u_{0}\right\|^{2} e^{2 \lambda_{\max } t} .
$$

Hence we get

$$
\left\|L\left[u_{0}\right](x, t)\right\| \leq e^{\lambda_{\max } t}\left\|u_{0}\right\|
$$


We will now state the main theorem on pattern formation under the fully nonlinear setting. We fix $\theta>0$ to be a small constant. For any small $\delta>0$ we define the escape time $T^{\delta}$ by

$$
\theta=\delta \exp \left(\lambda_{\max } T^{\delta}\right)
$$

The following theorem establishes a characterization of nonlinear patterns in the SH equation by a finite combination of the maximal linear growing modes.

Theorem 1. Let $u_{0} \in H^{2},\left\|u_{0}\right\|=1$. Then there exist constants $\delta_{0}>0, C>0, \theta>0$ depending on $\alpha$ such that for all $0<\delta \leq \delta_{0}$ if the initial pertubation of 0 is $u^{\delta}(x, 0)=\delta u_{0}$, then its nonlinear evolution $u^{\delta}(x, t)$ satisfies

$$
\left\|u^{\delta}(x, t)-\delta M\left[u_{0}\right](x, t)\right\| \leq C\left\{e^{-\nu t}+\delta\left\|u_{0}\right\|_{H^{2}}^{2}+\delta e^{\lambda_{\max } t}\right\} \delta e^{\lambda_{\max } t}
$$

for $0 \leq t \leq T^{\delta}$. Here

$$
M\left[u_{0}\right](x, t)=\left[\sum_{\mathbf{q} \in \Omega_{\max }} w_{\mathbf{q}} e_{\mathbf{q}}(x)\right] e^{\lambda_{\max } t} .
$$

Notice that Theorem 1 implies instability for the Swift-Hohenberg equations (1.1), (1.2), (1.3); refer to [4, 5].

The passage from linear to nonlinear instability does not rely on systematic machinery. In the PDE system with a dissipation, characterization of pattern formation has been developed in Kirchhoff ellipses [6], reaction-diffusion systems [5], and Keller-Segel systems [4. The methods in this paper are based on the bootstrap idea that was first introduced by Guo and Strauss.

In section 2, we will provide energy estimates to control the nonlinear terms in the $\mathrm{SH}$ equation and the nonlinear patterns will be established in section 3 .

2. Energy estimates. In this section, we provide energy estimates to control the nonlinear terms in the $\mathrm{SH}$ equation (1.1).

Lemma 2. If $u \in H^{4}\left(\mathbb{T}^{d}\right)$ satisfies (1.2), then for every derivative $|\partial| \leq 2$ we have

$$
\int_{\mathbb{T}^{d}}(\triangle \partial u)^{2} d x=\sum_{i=1}^{d} \int_{\mathbb{T}^{d}}\left(\partial u_{x_{i} x_{i}}\right)^{2}+2 \sum_{1 \leq i<j \leq d} \int_{\mathbb{T}^{d}}\left(\partial u_{x_{i} x_{j}}\right)^{2} .
$$

Here $u_{x_{i} x_{j}}=\frac{\partial^{2} u}{\partial x_{i} \partial x_{j}}$.

Proof. First of all, we note that

$$
(\triangle \partial u)^{2}=\left(\sum_{i=1}^{d} \partial u_{x_{i} x_{i}}\right)^{2}=\sum_{i=1}^{d}\left(\partial u_{x_{i} x_{i}}\right)^{2}+2 \sum_{1 \leq i<j \leq d} \partial u_{x_{i} x_{i}} \partial u_{x_{j} x_{j}} .
$$

So we have

$$
\int_{\mathbb{T}^{d}}(\triangle \partial u)^{2}=\sum_{i=1}^{d} \int_{\mathbb{T}^{d}}\left(\partial u_{x_{i} x_{i}}\right)^{2}+2 \sum_{1 \leq i<j \leq d} \int_{\mathbb{T}^{d}} \partial u_{x_{i} x_{i}} \partial u_{x_{j} x_{j}} .
$$


By the boundary condition (1.2) we can integrate by parts to obtain

$$
\begin{aligned}
\int_{\mathbb{T}^{d}} \partial u_{x_{i} x_{i}} \partial u_{x_{j} x_{j}} & =-\int_{\mathbb{T}^{d}} \partial u_{x_{i}} \partial u_{x_{j} x_{j} x_{i}} \\
& =-\int_{\mathbb{T}^{d}} \partial u_{x_{i}} \partial u_{x_{i} x_{j} x_{j}} \\
& =\int_{\mathbb{T}^{d}} \partial u_{x_{i} x_{j}} \partial u_{x_{i} x_{j}} \\
& =\int_{\mathbb{T}^{d}} \partial u_{x_{i} x_{j}}^{2} .
\end{aligned}
$$

Therefore we get

$$
\int_{\mathbb{T}^{d}}(\triangle \partial u)^{2} d x=\sum_{i=1}^{d} \int_{\mathbb{T}^{d}}\left(\partial u_{x_{i} x_{i}}\right)^{2}+2 \sum_{1 \leq i<j \leq d} \int_{\mathbb{T}^{d}}\left(\partial u_{x_{i} x_{j}}\right)^{2} .
$$

Lemma 3. If $u(x, t)$ is a solution of (1.1), (1.2), (1.3) satisfying $\|u(x, t)\|_{H^{2}} \leq \eta$, then we have

$$
\frac{1}{2} \frac{d}{d t} \sum_{|\partial|=2} \int_{\mathbb{T}^{d}}(\partial u)^{2} d x \leq C_{2}\|u\|^{2}
$$

for

$$
\begin{gathered}
C_{2}=\frac{\left(\alpha-1+2 d+d^{2} C_{1}\right)^{2}}{2} d^{2}, \\
C_{1}=C_{\eta}\left(1+C_{0}^{2} \eta\right),
\end{gathered}
$$

where $C_{0}$ is a universal constant.

Proof. Taking second order $\partial$ derivatives of (1.1) we have

$$
\begin{aligned}
\frac{d}{d t} \partial u & =\alpha \partial u-(1+\triangle)^{2} \partial u-\partial f(u) \\
& =(\alpha-1) \partial u-\triangle^{2} \partial u-2 \triangle \partial u-\partial f(u) .
\end{aligned}
$$

Hence we get

$$
\begin{aligned}
\frac{1}{2} \frac{d}{d t}|\partial u|^{2}= & (\alpha-1)|\partial u|^{2}-\left(\triangle^{2} \partial u\right) \partial u-2(\triangle \partial u) \partial u-\partial f(u) \partial u \\
\frac{1}{2} \frac{d}{d t} \int_{\mathbb{T}^{d}}|\partial u|^{2}= & (\alpha-1) \int_{\mathbb{T}^{d}}|\partial u|^{2}-\int_{\mathbb{T}^{d}}\left(\triangle^{2} \partial u\right) \partial u-2 \int_{\mathbb{T}^{d}}(\triangle \partial u) \partial u \\
& -\int_{\mathbb{T}^{d}} \partial f(u) \partial u \\
= & (\alpha-1) \int_{\mathbb{T}^{d}}|\partial u|^{2}-\int_{\mathbb{T}^{d}}|\triangle \partial u|^{2}+2 \int_{\mathbb{T}^{d}}|\nabla \partial u|^{2} \\
& -\int_{\mathbb{T}^{d}} \partial f(u) \partial u
\end{aligned}
$$

by integration by parts and using the boundary condition (1.2). We now treat the last nonlinear term $\int_{\mathbb{T}^{d}} \partial f(u) \partial u$. 
Assume $\partial=\frac{\partial^{2}}{\partial x_{i_{1}} \partial x_{i_{2}}}$.

$$
\partial f(u)=f^{\prime}(u) \partial u+f^{\prime \prime}(u) u_{x_{i_{1}}} u_{x_{i_{2}}} .
$$

Therefore we get

$$
\int_{\mathbb{T}^{d}} \partial f(u) \partial u=\int_{\mathbb{T}^{d}} f^{\prime}(u)(\partial u)^{2}+\int_{\mathbb{T}^{d}} f^{\prime \prime}(u) u_{x_{i_{1}}} u_{x_{i_{2}}} \partial u .
$$

By assumption (1.10) and $\|u(x, t)\|_{H^{2}} \leq \eta$,

$$
-\int_{\mathbb{T}^{d}} \partial f(u) \partial u \leq C_{\eta}\left[\int_{\mathbb{T}^{d}}|\partial u|^{2}+\|\nabla u\|_{L^{4}}\|\nabla u\|_{L^{4}}\left\|\nabla^{2} u\right\|\right] .
$$

We have a constant $C_{0}>0$ such that if $g \in H^{1}\left(\mathbb{T}^{d}\right)$ and $\int_{\mathbb{T}^{d}} g=0$, then

$$
\|g\|+\|g\|_{L^{4}} \leq C_{0}\|\nabla g\| .
$$

Hence we get $\|\nabla u\|_{L^{4}} \leq C_{0}\left\|\nabla^{2} u\right\|$ and $\|\nabla u\|_{L^{4}} \leq C_{0}\|u\|_{H^{2}} \leq C_{0} \eta$. Therefore we get

$$
-\int_{\mathbb{T}^{d}} \partial f(u) \partial u \leq C_{\eta}\left(1+C_{0}^{2} \eta\right)\left\|\nabla^{2} u\right\|^{2} .
$$

Let $C_{1}=C_{\eta}\left(1+C_{0}^{2} \eta\right)$; then

$$
-\int_{\mathbb{T}^{d}} \partial f(u) \partial u \leq C_{1}\left\|\nabla^{2} u\right\|^{2} .
$$

Set $v=\partial u$ :

$$
\int_{\mathbb{T}^{d}}|\nabla \partial u|^{2}=\int_{\mathbb{T}^{d}}|\nabla v|^{2}=\sum_{i=1}^{d} \int_{\mathbb{T}^{d}}\left|v_{x_{i}}\right|^{2}=-\sum_{i=1}^{d} \int_{\mathbb{T}^{d}} v v_{x_{i} x_{i}} .
$$

Hence we get

$$
\begin{aligned}
\int_{\mathbb{T}^{d}}|\nabla \partial u|^{2} & \leq \sum_{i=1}^{d}\left[\int_{\mathbb{T}^{d}} \frac{1}{4}\left|v_{x_{i} x_{i}}\right|^{2}+\int_{\mathbb{T}^{d}} v^{2}\right] \\
& \leq \frac{1}{4} \int_{\mathbb{T}^{d}}|\triangle \partial u|^{2}+d \int_{\mathbb{T}^{d}} v^{2}
\end{aligned}
$$

since $\sum_{i=1}^{d} \int_{\mathbb{T}^{d}}\left|v_{x_{i} x_{i}}\right|^{2} \leq \int_{\mathbb{T}^{d}}|\triangle \partial u|^{2}$ by Lemma 2, Therefore we have

$$
\begin{aligned}
(\alpha-1) \int_{\mathbb{T}^{d}}|\partial u|^{2}-\int_{\mathbb{T}^{d}}|\triangle \partial u|^{2}+2 \int_{\mathbb{T}^{d}}|\nabla \partial u|^{2} & \leq(\alpha-1+2 d) \int_{\mathbb{T}^{d}}|\partial u|^{2} \\
& -\frac{1}{2} \int_{\mathbb{T}^{d}}|\triangle \partial u|^{2} .
\end{aligned}
$$

From (2.1), (2.3), (2.4) we have

$$
\begin{aligned}
\frac{1}{2} \frac{d}{d t} \sum_{|\partial|=2} \int_{\mathbb{T}^{d}}|\partial u|^{2} \leq & (\alpha-1+2 d) \sum_{|\partial|=2} \int_{\mathbb{T}^{d}}|\partial u|^{2}-\frac{1}{2} \sum_{|\partial|=2} \int_{\mathbb{T}^{d}}|\triangle \partial u|^{2} \\
& +d^{2} C_{1}\left\|\nabla^{2} u\right\|^{2}
\end{aligned}
$$

Let $A_{1}=\alpha-1+2 d+d^{2} C_{1}$; then

$$
\frac{1}{2} \frac{d}{d t} \sum_{|\partial|=2} \int_{\mathbb{T}^{d}}|\partial u|^{2} \leq A_{1} \sum_{|\partial|=2} \int_{\mathbb{T}^{d}}|\partial u|^{2}-\frac{1}{2} \sum_{|\partial|=2} \int_{\mathbb{T}^{d}}|\triangle \partial u|^{2} .
$$


By Lemma 2] we have

$$
\int_{\mathbb{T}^{d}}|\triangle \partial u|^{2}=\sum_{i=1}^{d} \int_{\mathbb{T}^{d}}\left|\partial u_{x_{i} x_{i}}\right|^{2}+2 \sum_{1 \leq i<j \leq d} \int_{\mathbb{T}^{d}}\left|\partial u_{x_{i} x_{j}}\right|^{2} .
$$

Hence we get

$$
\begin{aligned}
\sum_{|\partial|=2} \int_{\mathbb{T}^{d}}|\triangle \partial u|^{2}=\sum_{|\partial|=4} \int_{\mathbb{T}^{d}}|\partial u|^{2} & , \\
\sum_{|\partial|=2} \int_{\mathbb{T}^{d}}|\partial u|^{2} & =\sum_{i=1}^{d} \sum_{j=1}^{d} \int_{\mathbb{T}^{d}} u_{x_{i} x_{j}}^{2} \\
& =\sum_{i=1}^{d} \sum_{j=1}^{d} \int_{\mathbb{T}^{d}} u u_{x_{i} x_{i} x_{j} x_{j}} \\
& \leq \frac{1}{2 A_{1}} \sum_{|\partial|=4} \int_{\mathbb{T}^{d}}|\partial u|^{2}+\frac{A_{1}}{2} d^{2} \int_{\mathbb{T}^{d}} u^{2} \\
& \leq \frac{1}{2 A_{1}} \sum_{|\partial|=2} \int_{\mathbb{T}^{d}}|\triangle \partial u|^{2}+\frac{A_{1}}{2} d^{2} \int_{\mathbb{T}^{d}} u^{2} .
\end{aligned}
$$

From (2.5) and (2.6) we have

$$
\frac{1}{2} \frac{d}{d t} \sum_{|\partial|=2} \int_{\mathbb{T}^{d}}|\partial u|^{2} d x \leq \frac{A_{1}^{2}}{2} d^{2} \int_{\mathbb{T}^{d}} u^{2} .
$$

This completes the proof.

Lemma 4. Suppose $u(x, t)$ is a solution of (1.1) such that $\|u(x, t)\|_{H^{2}} \leq \eta$ and

$$
\|u(x, t)\| \leq 2 e^{\lambda_{\max } t}\|u(x, 0)\| .
$$

Then we have

$$
\|u(t)\|_{H^{2}}^{2} \leq C_{3}\left\{\|u(0)\|_{H^{2}}^{2}+e^{2 \lambda_{\max } t}\|u(0)\|^{2}\right\},
$$

where $C_{3}=(2+\sqrt{d}) \max \left\{\frac{4 C_{2}}{\lambda_{\max }}, 4\right\}$.

Proof. We first consider the second-order derivatives of $u(x, t)$. From the previous lemma and our assumptions on $\|u(t)\|_{H^{2}} \leq \eta$ we have

$$
\frac{1}{2} \frac{d}{d t} \sum_{|\partial|=2} \int_{\mathbb{T}^{d}}|\partial u|^{2} d x \leq C_{2}\|u(t)\|^{2} \leq 4 C_{2} e^{2 \lambda_{\max } t}\|u(0)\|^{2} .
$$

Integrating (2.9) from 0 to $t$ we have

$$
\begin{aligned}
\sum_{|\partial|=2} \int_{\mathbb{T}^{d}}|\partial u(x, t)|^{2} d x & \leq \sum_{|\partial|=2} \int_{\mathbb{T}^{d}}|\partial u(x, 0)|^{2} d x+\frac{4 C_{2}}{\lambda_{\max }} e^{2 \lambda_{\max } t}\|u(0)\|^{2} \\
& \leq\|u(0)\|_{H^{2}}^{2}+\frac{4 C_{2}}{\lambda_{\max }} e^{2 \lambda_{\max } t}\|u(0)\|^{2} .
\end{aligned}
$$

For the $0^{\text {th }}$ and the first derivatives:

$$
\|u(t)\|^{2} \leq 4 e^{2 \lambda_{\max } t}\|u(0)\|^{2}
$$


by (2.8).

$$
\begin{aligned}
\sum_{j=1}^{d} \int_{\mathbb{T}^{d}}\left(\frac{\partial u}{\partial x_{j}}\right)^{2} & =-\sum_{j=1}^{d} \int_{\mathbb{T}^{d}} u \frac{\partial^{2} u}{\partial x_{j}^{2}} \\
& \leq \frac{\sqrt{d}}{2} \sum_{j=1}^{d} \int_{\mathbb{T}^{d}}\left(\frac{\partial^{2} u}{\partial x_{j}^{2}}\right)^{2}+\frac{1}{2 \sqrt{d}} \sum_{j=1}^{d} \int_{\mathbb{T}^{d}} u^{2} \\
& \leq \frac{\sqrt{d}}{2} \sum_{|\partial|=2} \int_{\mathbb{T}^{d}}|\partial u|^{2} d x+\frac{\sqrt{d}}{2} \int_{\mathbb{T}^{d}} u^{2}
\end{aligned}
$$

Thus the lemma follows.

\section{Proof of the main result.}

Proof. Let $u^{\delta}(x, t)$ be a family of solutions to the SH equation (1.1) with initial data $u^{\delta}(x, 0)=\delta u_{0}$. Define

$$
\begin{gathered}
T^{*}=\sup \left\{t>0 \mid\left\|u^{\delta}(s)-\delta L\left[u_{0}\right](., s)\right\| \leq \frac{\delta}{2} e^{\lambda_{\max } s}, \forall 0 \leq s \leq t\right\}, \\
T^{* *}=\sup \{t>0 \mid\|u(s)\| \leq \eta, \forall 0 \leq s \leq t\} .
\end{gathered}
$$

By the definition of $T^{*}$ for $0 \leq t \leq T^{*}$ and by Lemma 1, we get

$$
\left\|u^{\delta}(t)\right\| \leq \frac{3}{2} \delta e^{\lambda_{\max } t} .
$$

Using Lemma 4 we have

$$
\left\|u^{\delta}(t)\right\|_{H^{2}} \leq \sqrt{C}_{3}\left\{\delta\|u(0)\|_{H^{2}}+\delta e^{\lambda_{\max } t}\right\} .
$$

We estimate the $L^{2}$ norm of $u^{\delta}(x, t)$ for $0 \leq t \leq \min \left\{T^{\delta}, T^{*}, T^{* *}\right\}$. Apply Duhamel's principle to obtain

$$
u^{\delta}(t)=\delta L\left[u_{0}\right](., t)+\int_{0}^{t} L\left[-f\left(u^{\delta}(\tau)\right)\right](., t-\tau) d \tau .
$$

Apply Lemma 1 to deduce

$$
\left\|u^{\delta}(t)-\delta L\left[u_{0}\right](., t)\right\| \leq \int_{0}^{t} e^{\lambda_{\max }(t-\tau)} \| f\left(\left(u^{\delta}(\tau)\right) \| d \tau .\right.
$$

Since $f(0)=f^{\prime}(0)=0$ there exists $v(x, \tau) \in \mathbb{R}$ with $|v(x, \tau)| \leq\left|u^{\delta}(x, \tau)\right|$ such that

$$
f\left(u^{\delta}(x, \tau)\right)=\frac{f^{\prime \prime}(v(x, \tau))}{2} u^{\delta}(x, \tau)^{2} .
$$

By $\left\|u^{\delta}(\tau)\right\|_{H^{2}} \leq \eta$ from (1.9) we have $\left\|u^{\delta}(x, \tau)\right\|_{\infty} \leq C_{0} \eta$ and this implies $\left|v^{\delta}(x, \tau)\right| \leq$ $C_{0} \eta$. By (1.10) we have

$$
\left|f^{\prime \prime}(v(x, \tau))\right| \leq C_{\eta}
$$

so we get

Hence we deduce

$$
\left|f\left(u^{\delta}(x, \tau)\right)\right| \leq \frac{1}{2} C_{\eta} u^{\delta}(x, \tau)^{2} .
$$

$$
\left\|f\left(u^{\delta}(\tau)\right)\right\| \leq \frac{1}{2} C_{\eta}\left\|u^{\delta}(\tau)\right\|\left\|u^{\delta}(\tau)\right\|_{\infty} .
$$


By (1.9) we have

$$
\left\|f\left(u^{\delta}(\tau)\right)\right\| \leq \frac{1}{2} C_{\eta}\left\|u^{\delta}(\tau)\right\|_{H^{2}} C_{0}\left\|u^{\delta}(\tau)\right\|_{H^{2}}=\frac{C_{0} C_{\eta}}{2}\left\|u^{\delta}(\tau)\right\|_{H^{2}}^{2}
$$

From (3.2) and (3.3) we have

$$
\left\|u^{\delta}(t)-\delta L\left[u_{0}\right](., t)\right\| \leq \frac{C_{0} C_{\eta}}{2} \int_{0}^{t} e^{\lambda_{\max }(t-\tau)}\left\|u^{\delta}(\tau)\right\|_{H^{2}}^{2} d \tau .
$$

By Lemma 4 we get

$$
\left\|u^{\delta}(\tau)\right\|_{H^{2}}^{2} \leq C_{3}\left\{\delta^{2}\|u(0)\|_{H^{2}}^{2}+\delta^{2} e^{2 \lambda_{\max } \tau}\right\} .
$$

Therefore we obtain

$$
\begin{aligned}
\| u^{\delta}(t)- & \delta L\left[u_{0}\right](., t) \| \\
& \leq \frac{C_{0} C_{\eta} C_{3}}{2} \int_{0}^{t} e^{\lambda_{\max }(t-\tau)}\left\{\delta^{2}\|u(0)\|_{H^{2}}^{2}+\delta^{2} e^{2 \lambda_{\max } \tau}\right\} d \tau \\
& \leq \frac{C_{0} C_{\eta} C_{3}}{2}\left\{\frac{\delta\|u(0)\|_{H^{2}}^{2}}{\lambda_{\max }}+\frac{1}{\lambda_{\max }} \delta e^{\lambda_{\max } t}\right\} \delta e^{\lambda_{\max } t}
\end{aligned}
$$

We choose $\theta>0$ and $\delta_{0}>0$ such that

$$
\begin{gathered}
2 \sqrt{C_{3}} \theta<\eta \\
\frac{C_{0} C_{\eta} C_{3}}{2} \theta<\frac{\lambda_{\max }}{4}, \\
\delta_{0}\left\|u_{0}\right\|_{H^{2}} \leq \theta \\
\frac{C_{0} C_{\eta} C_{3}}{2} \delta_{0}\left\|u_{0}\right\|_{H^{2}}^{2}<\frac{\lambda_{\max }}{4} .
\end{gathered}
$$

Then we will prove by contradiction that for $0<\delta \leq \delta_{0}$ we have

$$
T^{\delta}=\min \left\{T^{\delta}, T^{*}, T^{* *}\right\}
$$

and our theorem follows.

Case 1) Suppose that $T^{* *}$ is minimum. We can let $t=T^{* *}$ in (3.1) to get

$$
\begin{aligned}
\left\|u^{\delta}\left(T^{* *}\right)\right\|_{H^{2}} & \leq \sqrt{C}_{3}\left\{\delta\|u(0)\|_{H^{2}}+\delta e^{\lambda_{\max } T^{\delta}}\right\} \\
& \leq \sqrt{C}_{3}\left\{\delta\|u(0)\|_{H^{2}}+\theta\right\} \\
& \leq 2 \sqrt{C}_{3} \theta
\end{aligned}
$$

by our choice of $\delta_{0}$ in (3.7). By the choice of $\theta$ in (3.5):

$$
\left\|u^{\delta}\left(T^{* *}\right)\right\|_{H^{2}}<\eta
$$

This is a contradiction to the definition of $T^{* *}$. 
Case 2) Suppose that $T^{*}$ is the smallest in $\left\{T^{\delta}, T^{*}, T^{* *}\right\}$. We let $t=T^{*}$ in (3.4) to get

$$
\begin{aligned}
\| u^{\delta}\left(T^{*}\right)- & \delta L\left[u_{0}\right]\left(., T^{*}\right) \| \\
& \leq \frac{C_{0} C_{\eta} C_{3}}{2}\left\{\frac{\delta\|u(0)\|_{H^{2}}^{2}}{\lambda_{\max }}+\frac{1}{\lambda_{\max }} \delta e^{\lambda_{\max } T^{\delta}}\right\} \delta e^{\lambda_{\max } T^{*}} \\
& \leq \frac{C_{0} C_{\eta} C_{3}}{2}\left\{\frac{\delta\|u(0)\|_{H^{2}}^{2}}{\lambda_{\max }}+\frac{1}{\lambda_{\max }} \theta\right\} \delta e^{\lambda_{\max } T^{*}} .
\end{aligned}
$$

For $0<\delta \leq \delta_{0}$ by our choice of $\theta, \delta_{0}$ in (3.8) and (3.6) we have

$$
\left\|u^{\delta}\left(T^{*}\right)-\delta L\left[u_{0}\right]\left(., T^{*}\right)\right\|<\frac{\delta}{2} e^{\lambda_{\max } T^{*}} .
$$

This contradicts the definition of $T^{*}$. Thus this completes the proof.

Acknowledgement. H. J. Hwang is supported by the National Research Foundation of Korea (NRF) funded by the Ministry of Education, Science and Technology (Grant 2010-0008127).

\section{REFERENCES}

[1] G. Caginalp and P.C. Fife, Higher order phase field models and detailed anisotropy. Phys. Rev. B 34 (1986), 4940-4943. MR875362 (88f:82019)

[2] M.C. Cross and P.C. Hohenberg, Pattern formation outside of equilibrium. Rev. Mod. Phys. 65 (1993), 851-1112.

[3] P. Grinrod, Patterns and Waves: The Theory and Applications of Reaction-Diffusion Equations, Oxford: Clarendon, 1991. MR.1136256 (92k:35145)

[4] Y. Guo, H.J. Hwang, Pattern formation. I: The Keller-Segel model, J. Differential Equations 249 (2010), 1519-1530.

[5] Y. Guo and H.J. Hwang, Pattern formation. II. The Turing instability, Proc. Amer. Math. Soc. 135 (2007), 2855-2866. MR2317962 (2008a:35131)

[6] Y. Guo, C. Hallstrom, and D. Spirn, Dynamics near an unstable Kirchhoff ellipse, Comm. Math. Phys. 245 (2004), 297-354. MR2039699 (2005m:76039)

[7] H-J. Hwang and Y. Guo, On the dynamical Rayleigh-Taylor instability. Arch. Ration. Mech. Anal. 167 (2003), no. 3, 235-253. MR1978583 (2004f:76064)

[8] J. Swift and P.C. Hohenberg, Hydrodynamic fluctuations at the convective instability, Phys. Rev. A 15 (1977), 319.

[9] R.E. La Quey, P.H. Mahajan, P.H. Rutherford, and W.M. Tang, Phys. Rev. Lett. 34 (1975), 391.

[10] T. Shang and G.L. Sivashinsky, Phys. J. 43 (1983), 459-466.

[11] L.A. Peletier and V. Rottschäfer, Pattern selection of solutions of the Swift-Hohenberg equation, Physica D 194 (2004), 95-126. MR2075667 (2005h:35176)

[12] L.A. Peletier and V. Rottschäfer, Large time behavior of solutions of the Swift-Hohenberg equation, C. R. Acad. Sci. Paris Ser. I Math. 336 (2003), 225-230. MR1968263 (2004b:35167)

[13] L.A. Peletier and J.F. Williams, Some canonical bifurcations in the Swift-Hohenberg equation, SIAM J. Appl. Dyn. Syst. 6 (2007), no. 1, 208-235. MR.2299639 (2007m:34051) 